\title{
Production of Recombinant LSC Protein in Nicotiana tabacum Hairy Root: Direct vs. Indirect, a Comparison
}

\author{
Ali Fallah ${ }^{1}$, Asal Akhaviann ${ }^{1}$, Rouhollah Kazemi ${ }^{1}$, Mahyat Jafari ${ }^{1}$, Ali-Hatef Salmanian ${ }^{1 *}$ \\ ${ }^{1}$ Department of Agricultural Biotechnology, National Institute of Genetic Engineering and Biotechnology (NIGEB), Tehran, Iran
}

Corresponding Author: Ali-Hatef Salmanian, Department of Agricultural Biotechnology, National Institute of Genetic Engineering and Biotechnology (NIGEB), Tehran, Iran. Tel: +98-21-44787365, Email: salman@nigeb.ac.ir

Received December 10, 2017; Revised February 9, 2018; Accepted February 15, 2018; Online Published March 30, 2018

\begin{abstract}
Introduction: The production and purification cost of a recombinant protein in plants is lower than other conventional systems, such as bacteria. Comparing different parts of the plant, the hairy root is known to be a suitable bioreactor to produce recombinant proteins including vaccine candidate immunogens. Among the most important causes of diarrhea, E. coli and Vibrio cholerae cause the disease by producing a toxin. The B subunit of this toxin is an appropriate candidate for vaccine development because of its non-toxicity and exposure to the immune system.

Materials and Methods: To investigate the efficiency of transgenic hairy roots in the production of recombinant protein, two methods were considered: direct (induction of hairy root with recombinant Agrobacterium rhizogenes) and indirect (production of transgenic plant with Agrobacterium tumefaciens and then polluting it with non-transgenic A. rhizogenes). To compare the 2 methods, GUS protein expression was used as a reporter and chimeric protein LSC (consisting of $I t B-s t \times B-c t \times B$ ) as an antigenic protein. Transformation of tobacco hairy root was confirmed by genomic Polymerase chain reaction (PCR). Gene expression comparison was investigated by semi-quantitative ELISA and enzymatic activity. Results: The results show that the activity of GUS reporter enzyme in the indirect method is seven times more than that of the direct method. Likewise, the expression of LSC recombinant protein in the indirect transformation was 1.5 times more than in direct method.

Conclusions: Comparing of these two methods indicated that the hairy roots in the indirect method yield higher protein content than in the direct method.

Keywords: Hairy Root, Agrobacterium Rhizogenes, Direct and Indirect Transformation

Citation: Fallah A, Akhavian A, Kazemi R, Jafari M, Salmanian AH. Production of recombinant LSC protein in Nicotiana tabacum hairy root: direct vs. indirect, a comparison. J Appl Biotechnol Rep. 2018;5(1):1-7. doi:10.29252/jabr.01.01.01.
\end{abstract}

\section{Introduction}

Plants have a high capability in production of various recombinant proteins, such as vaccines and enzymes. Among their advantages over other systems are affordability, easy transfer of research findings to production stages, capability of producing multipartite proteins, not contamination of plants with human pathogens, and their safety. ${ }^{1}$ Moreover, plant cells can be cultured under simple conditions and new products can be easily accumulated in target organelles such as the endoplasmic reticulum, protein storage vacuoles and plastids away from proteases. ${ }^{2}$ Due to the presence of mucosal immune system in the body of animals, in the case of the utilization of transgenic plant, it can be expected that these organisms will be immunized against the plant recombinant protein. ${ }^{1}$ Among different plant tissues hairy root is considered as an efficient bioreactor because of the advantages such as high protein production ability with the possibility of cultivation in industrial fermenters without the need for light and expensive hormones. ${ }^{3,4}$ The production and development of a successful vaccine against human and animal pathogens in hairy roots require selecting correct immunogens and appropriate designing of the synthetic genes for proper expression in the plant system. The bacteria causing intestinal infections and diarrheal disease, which is one of the major causes of death in children under-5 years, are good candidates for studying this system and selection of proper immunogens. ${ }^{5}$ The most important bacterial factors causing diarrheal infections are enterohemorrhagic E. coli (EHEC), enterotoxigenic E. coli (ETEC), and Vibrio cholerae that are major contributors to the development of endemic and epidemic diarrhea around the world. ${ }^{6,7}$ The toxin produced by these bacteria is the main factor causing diarrhea. The toxin of enterohemorrhagic $E$. coli (EHEC) is similar to the Shigella dysentery toxin named Stx. ${ }^{8}$ The ETEC bacteria produce a heat-labile (LT) toxin and the Vibrio bacteria produce Cholera enterotoxin (Ctx). These toxins produce diarrhea by causing disorders in the mechanism of ion channels of intestinal cells. ${ }^{9,10}$ All these three toxins (LT, Stx, and Ctx) are members of the AB5 toxin family. In this group of toxins, the same pentameric B subunit mediates the toxin binding to the host cell surface receptor. ${ }^{11}$ Considering the structure of AB5 toxins, B subunits, as the binding subunit, is the best candidate for vaccine development, which is expected to prevent their operation by blocking the toxinreceptor binding. On the other hand, the B subunit of both LT

Copyright (C) 2018 The Author(s). This is an open-access article distributed under the terms of the Creative Commons Attribution License (http:// creativecommons.org/licenses/by/4.0), which permits unrestricted use, distribution, and reproduction in any medium, provided the original work is properly cited. 
and Ctx toxins have an adjuvant activity which can activate the immune system, then their use in a recombinant protein will simultaneously enhance this effect. ${ }^{12}$ In previous studies, the binding (B) subunit is known as a strong immunogen that is capable of producing an appropriate blood and mucosal immune response in an animal model. ${ }^{13-16}$ There has not been yet any research reported on the production of a chimeric protein including the binding subunits of all three toxins or their binary combination in the hairy root system. In our previous study, a three-partit gene construct containing the binding subunit of ltB-stxB-ctxB (LSC) after bioinformatics studies was synthesized. ${ }^{17}$ Here, the expression ability of this chimeric gene in transgenic hairy roots of tobacco plant was investigated by direct and indirect transformation. Expression measurement of GUS enzyme and LSC chimeric protein in the tobacco hairy roots was conducted with the aim of producing an oral vaccine based on hairy roots and comparing two direct and indirect methods in transgenic hairy roots as a new method. The performing stages of this study are presented as the following (Figure 1).

\section{Materials and Methods}

Preparation of pBI121-lsc Gene Construct

The $l s c$ gene construct was synthesized according to the previous studies. ${ }^{17}$ In order to clone the $l s c$ gene construct, the pUC57 vector containing this synthetic gene and the pBI121 vector were digested with $\mathrm{XbaI} / \mathrm{SacI}$ restriction enzymes. The ligation of the $l s c$ to $\mathrm{pBI} 121$ was performed and the construct was transformed to 2 strains of Agrobacterium by freezing and thawing. ${ }^{18}$ The stctxPF primer:

5'CCATACTCTAGAAAA ACAATGGCTGAT TGTGCTAAG3' and stctxPR: 5'GAATTCGAGCTCTCACAATTCATCCTTCTC3' were used to confirm the transformation of Agrobacterium. MCAMF primers: 5'CTATCCTCCGCAAGACCCTTCCTC3' that are located on CaMV35s

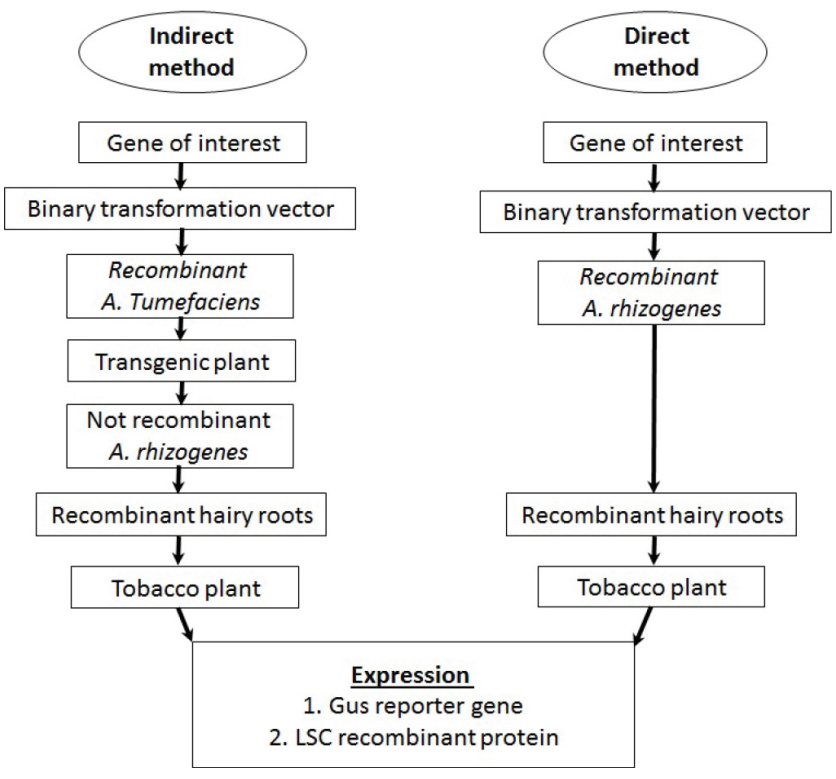

Figure 1. A Schematic Diagram of the Direct and Indirect Transformation Methods. promoter and MPLBR1 primer: 5' GAAGGTGCAAGCTACCTCTCT3' located on the left border of pBI121 were used to confirm the cloning and sequencing of the cloned genes in pBI121. Agrobacterium rhizogenes and Agrobacterium tumefaciens containing $\mathrm{pBI} 121$-gus and $\mathrm{pBI} 121$-lsc gene constructs were used for transformation of tobacco.

Transgenic Tobacco Plant With Recombinant Agrobacterium rhizogenes (Direct Method)

Using standard methods, tobacco seeds were cultured in sterile conditions and explants were prepared from leaves of the tobacco and their transformation was performed. ${ }^{19}$ After the emergence of hairy roots, every 7 days, one $3-4 \mathrm{~cm}$ slice from end of root from each line was transferred to a new selective medium with a higher concentration of kanamycin (75 and $100 \mathrm{mg} / \mathrm{L}$, respectively). After the final selection, roots were transferred to a liquid MS medium without antibiotic in Erlenmeyer flask at $24^{\circ} \mathrm{C}$ and $140 \mathrm{rpm}$ for further growth.

Transformation of Transgenic Tobacco With Nonrecombinant Agrobacterium rhizogenes (Indirect Method) In the indirect method, for the formation of hairy roots, first, the transgenic tobacco plant was obtained using A. tumefaciens LBA4404 containing the pBI121 + lsc gene construct and pBI121-gus plasmid. ${ }^{20}$ After evaluation of transgenic plants, leaf explants were prepared and hairy root was obtained using A. rhizogenes strain ATCC-15834. ${ }^{19}$

\section{Transgenic Plant and Hairy Roots Confirmation}

Genomic DNA was extracted from tissue samples of the leaves and hairy roots of plants. ${ }^{21}$ In order to confirm the presence of the recombinant $(l s c)$ and reporter $(g u s)$ gene, PCR reaction with $l s c$ gene and construct specific primer of the pBI121 vector was carried out. To ensure the presence of effective genes in the formation of transgenic hairy roots, rolBF: 5'GCTCTTGCAGTGCTAGATTTG3' and rolBR: 5'GAAGGTGCAAGCTACCTCTCT3' primers, which are specific for the rolB gene, were used in PCR reaction.

\section{Gus Assay}

The $20 \mathrm{mg} / \mathrm{mL}$ solution of X-Gluc (Fermentas) in DMSO (Merck) was formulated according to Terada et al. ${ }^{22}$ The qualitative study of GUS expression was performed in leaf and hairy root samples of transgenic plants and samples were photographed macroscopically. Also, the quantitative assay of gus in the cellular extracts of transgenic plants' tissues was measured according to Blazquez. ${ }^{23}$ After 10 and 20 minutes, the reaction was stopped by using $1 \mathrm{M}$ sodium carbonate solution, and the created color was measured at $455 \mathrm{~nm}$. The activity rate of gus in the samples was calculated based on the standard curve drawn for the 4-MUG.

Evaluation of the Recombinant Proteins Expressed in the Culture Medium of Transgenic Hairy Roots Quantitative ELISA was used to determine the amount of LSC protein in the total extracted protein. In this regard, a serial dilution of $10 \mathrm{pg}$ to $5 \mu \mathrm{g}$ of the purified protein LSC and the antibody against this protein was prepared. ${ }^{17}$ The endobiotic 
peroxidation activity in root tissues was stopped using $0.3 \%$ hydrogen peroxide. A protein solution from not-transformed hairy roots was used as negative control. The protein amount in the hairy root tissues of tobacco was calculated by processing the results obtained from the standard curve which was drawn based on the pure protein LSC.

Ethical Considerations

This study was approved by the ethics committee of the National Institute of Genetic Engineering and Biotechnology with IR.NIGEB.EC.1394.8.10 code.

\section{Results}

Cloning the $l s c$ Gene in the pBI121 Plasmid and Transformation to Agrobacterium

The pBI121 vector containing $l s c$ gene was extracted from $E$. coli DH5a bacteria and evaluated with XbaI and SacI enzymes digestion (Figure 2A). The results of the colony PCR with specific primers of recombinant genes on $A$. rhizogenes and A. tumefaciens colonies represents the 650-bp (Figure 2B) and $2200 \mathrm{bp}$ (Figure 2C) bands of the $l s c$ and gus genes in correct sizes.

Tissue Culture and Transgenesis of Tobacco Plant

The tobacco plant was transformed using A. rhizogenes containing the constructs (Figure 3). About 7-10 days after transformation of tobacco with recombinant $A$. rhizogenes, the roots began to develop and their growth continued in the medium containing kanamycin (Figure 3A). The resistant single roots were transferred to solid culture medium with higher concentrations of kanamycin (Figure 3B). Roots were sub-cultured every 10 days by transferring one piece of root to the new medium, and then each line was transferred to the Erlenmeyer for further growth (Figure 3D). The remarkable point in the hairy roots of tobacco was their direct regeneration to seedlings and then the complete plant, as shown in Figure 3C. These seedlings became a complete plant, and also developed reproductive and seed production stages.

Various stages of tobacco transformation by A. tumefaciens are represented in Figure 4. In order to develop hairy roots, the leaf of transgenic plants, after validation of transformation, was exposed to non-transgenic $A$. rhizogenes.

\section{Genomic PCR of Transgenic Hairy Roots}

DNA was extracted from leaves and hairy roots of transgenic plants grown in the selected medium. The result of PCR with primers specific for $l s c$ and gus genes confirms transgenic plants at the genome level. The amplification of the $636 \mathrm{bp}$ segment with stctPF and stctPR primers confirms the $l s c$ gene transfer to the genome of transgenic tobacco plants (Figure $5 \mathrm{~B})$. Using the MCAMF (located in the promoter region) and stctPR primers in the PCR reaction for the $l s c$ gene, the amplification of 1106 bp segment is observable (Figure 5A). Using the pair primers of MCAMF/MPLBR1 for transgenic samples with gus genes resulted in the proliferation of

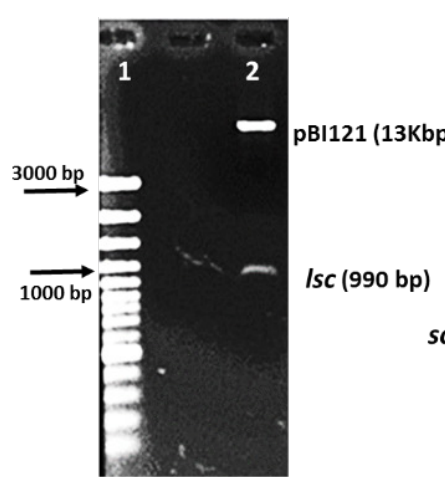

A

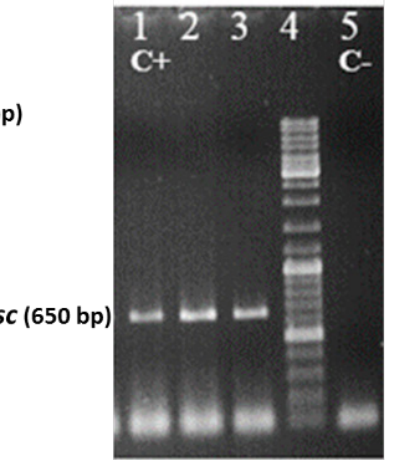

B

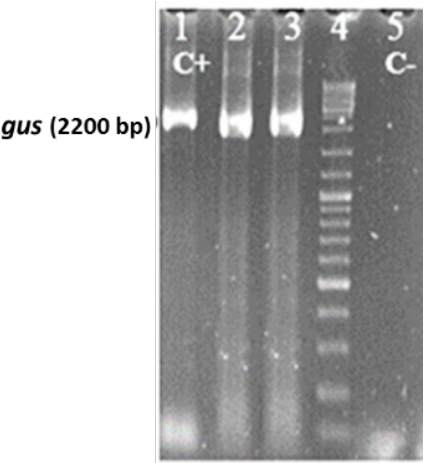

C

Figure 2. (A) Electrophoresis of the enzymatic digestion of pBI121-Isc recombinant vectors on Agarose gel 1\%, 1) DNA molecular marker, 2) recombinant $\mathrm{pBI}-$ IsC vector cut with Xbal and Sacl enzymes, (B) Electrophoresis of the PCR product of Agrobacterium rhizogenes ATCC15834 strain colonies transformed by pBI121 + IsC, and (C) pBI121 + gus on the 1\% Agarose gel, the molecular size marker was the Mix (Fermentas). C+ : positive control using pUC57 vector containing LSC construct, C- : negative control, the product of PCR without DNA template, 2 and 3) PCR product of two different colonies of Agrobacterium rhizogenes.
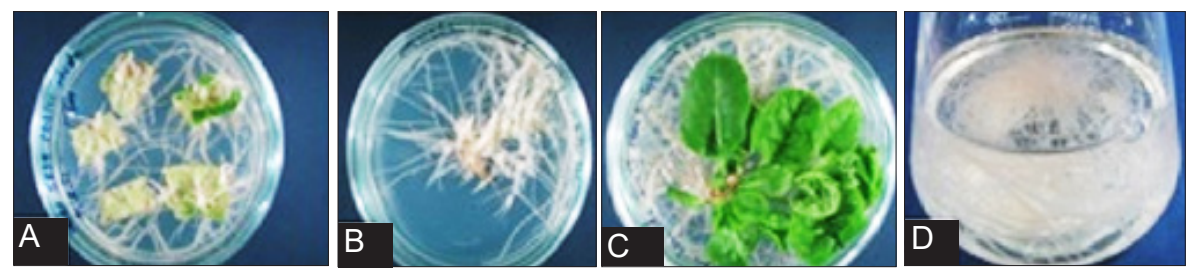

Figure 3. Transformation of Tobacco by Agrobacterium rhizogenes. (A) formation of hairy roots in solid culture medium, (B) development of a line of hairy roots in the solid medium, (C) formation of seedling from hairy roots, and (D) growing hairy roots in Erlenmeyer flask in a liquid culture medium. 


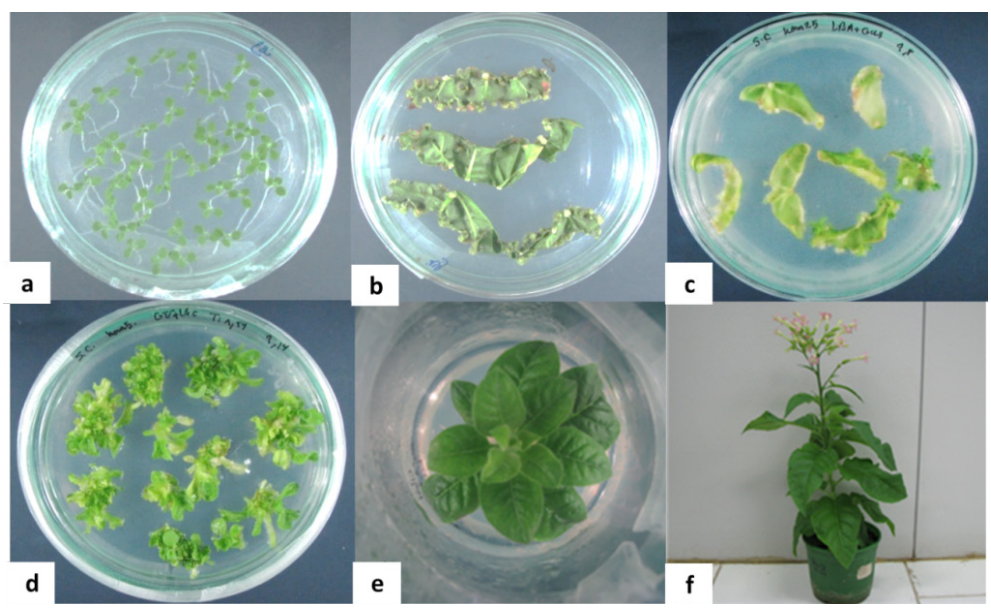

Figure 4. Different Stages of Transformation of Tobacco by Agrobacterium tumefaciens. (a) Seed culture, (b) Initial calluses on leaf explants, (c) and (d) Callus regeneration, (e) Development of transgenic plants, and (f) Reproductive stage of the transgenic tobacco.

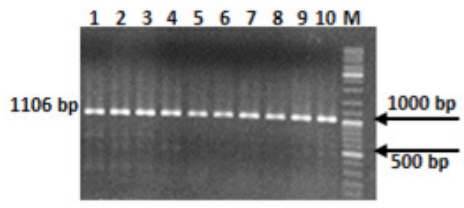

A

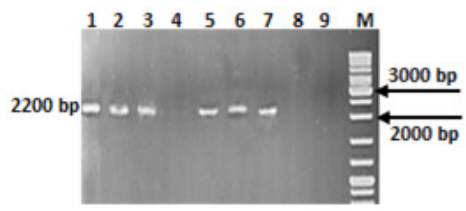

C

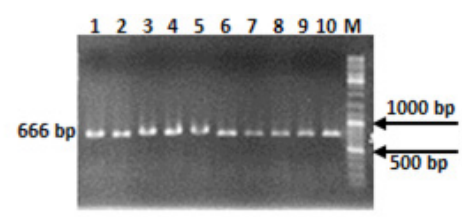

B

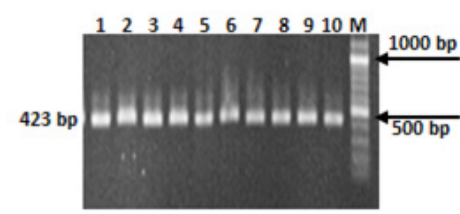

D

Figure 5. Molecular Confirmation of Hairy Roots by PCR Method. The PCR product using the genome of transgenic tobacco root containing Isc gene by MCAMF and stctPR primers (A), and the stctPF and stctPR primers (B), 1 to 5) transgenic hairy root of direct method and 6 to 10) transgenic hairy root of indirect method; PCR product of transgenic tobacco containing gus gene by MCAMF and MPLBR1 primers (C); PCR product of transgenic hairy roots using rolBf and rolBR primers (D), 1 to 5 ) hairy root of direct method and 6 to 10 ) transgenic hairy root by indirect method.

$2200 \mathrm{bp}$ segment in these samples (Figure 5C). To confirm transferring the rolB gene of $A$. rhizogenes involved in rooting, the PCR reaction was performed by rolBF and rolBR primers and resulted in the observing of the $423 \mathrm{bp}$ band related to the rolB gene (Figure 5D).

Quantitative and Histochemical Evaluation of GUS in Transgenic Samples

The GUS assay was performed on leaf and hairy roots of transgenic plants. In this evaluation, blueness was observed in leaf tissues of leaves and roots of the transgenic plants, but not in the control plant (Figure 6). The qualitative study of the leaves of the transgenic plant that was used in the indirect method represents the expression of GUS protein. In the qualitative comparison of hairy root between the direct transgenic method (Figure 6C) and indirect transgenic method (Figure 6B), it seems that GUS protein was more produced in the root tissue with indirect transgenesis, as the color is observed throughout the hairy root, but in the hairy root of the direct method it was seen as scattered spots on some points of the root. However, in the hairy root in which the gus gene was not transferred, no blue color was observed at all (Figure 6D).

In order to compare the direct and indirect methods in the hairy roots production and the gene expression, the quantitative study of GUS enzyme activity was conducted. The result of this evaluation is presented in a column diagram (Figure 7), which shows hairy root produced in the indirect method had more enzymatic activity. Accordingly, the average GUS activity in the ten lines of examined hairy root for the indirect method after 10 minutes was $326 \mathrm{nmol} / \mathrm{min}$, while for the same number of transgenic hairy root of direct method was $41 \mathrm{nmol} / \mathrm{min}$. A similar trend is observed with a decrease in GUS activity in 20 minutes after starting the enzyme measurement (Figure 7).

Evaluation of Recombinant Protein Production content in Transgenic Plants

ELISA was used for measuring LSC protein in hairy roots and its culture medium, and the percentage of LSC protein from 

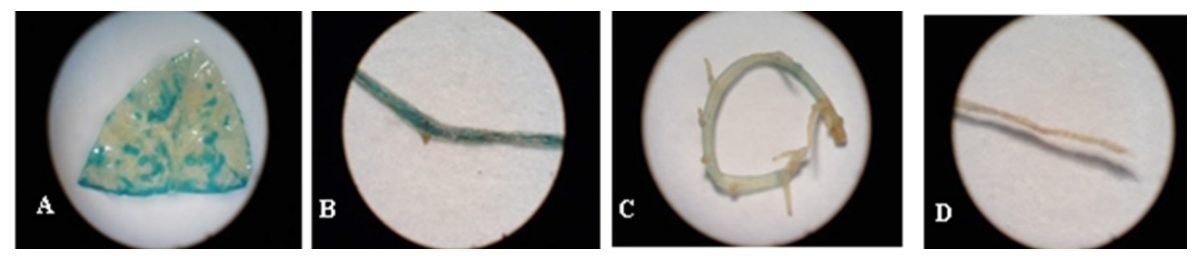

Figure 6. GUS Assay in Tissue of Tobacco Plant. (A) The leaves of transgenic plants with Agrobacterium tumefaciens containing pBI121 + gus, (B) The hairy root obtained from indirect method, (C) The hairy root resulted from direct method, (D) The transgenic sample transformed by Agrobacterium rhizogenes containing Isc + pBI121 (negative control).

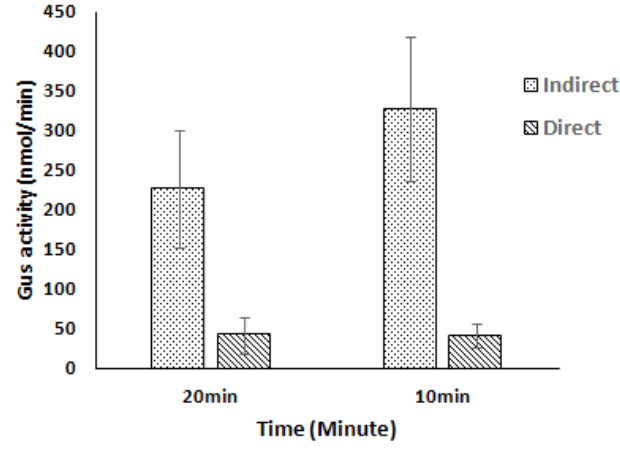

Figure 7. Comparison of GUS Enzyme Activity in Hairy Roots of Tobacco Plant Resulted From Direct and Indirect Methods.

total soluble proteins was calculated. ${ }^{17}$ The results showed that the average amount of LSC produced in the direct method in the hairy roots was $4 / 5 \mu \mathrm{g} / \mathrm{g} \mathrm{Fw}(0.13 \%$ of TSP), whereas this value was $5.5 \mu \mathrm{g} / \mathrm{g} \mathrm{Fw}(0.24 \%$ of TSP) in the indirect method. The amount of secreted LSC in the culture medium of hairy roots in the direct method was observed to be $1.1 \mu \mathrm{g} / \mathrm{g} \mathrm{Fw}$ $1.0(0.1 \%$ of TSP) and in the indirect method was $1.87 \mu \mathrm{g} / \mathrm{g}$ Fw $(0.17 \%$ of TSP). The sampling was performed in a 35 -day period and the results are shown in Figure 8. The diagram of Figure 8A shows that the intracellular expression of the LSC protein in the indirect method is greater than the direct method. Similar result is observed for the secreted protein in the diagram of Figure 8B.

\section{Discussion}

Plants, as a biological reactor, are exploited in the mass production of vaccines, antibodies, protein drugs, enzymes, and biological polymers for medical and industrial purposes. ${ }^{2}$ Among different parts of the plant, hairy roots are of great interest because of their advantages such as ability to produce high biomass in a relatively short time, genetic stability, and no need for expensive hormone and culture medium. ${ }^{4,25}$ In this study, the simultaneous transferring of T-DNAs (from $\mathrm{Ri}$ and pBI121 plasmid) in one step (direct transformation) or two-step (indirect transformation) was investigated. The hairy root was also used to express a vaccine candidate (LSC), a triple gene construct including the binding domain of heat sensitive toxin (LT) of ETEC bacteria, Shiga-like toxin (STX) of EHEC bacteria, and Cholera toxin (CTX) of Vibrio cholerae.

The required time for rooting in the direct method was about 8 days and in the indirect method was about 5 days. The reason for this difference may be the presence of kanamycin antibiotics in rooting medium of the direct method. ${ }^{26}$ Another observation was that better rooting cases were obtained from larger leaves than the smaller ones. It may be due to the releasing phenolic compounds found in the cut site, and also the secretion of the compounds such as glucose and $\mathrm{pH}$ lowering agents from the vacuoles which, according to the mechanism of gene transfer by Agrobacterium, ${ }^{27}$ can stimulate cells to receive external DNA more efficiently. Therefore, larger leaves seem to be more susceptible for rooting due to more material accumulation in their vacuoles.

Investigating the growth of hairy roots in the culture medium by gradual increase of the antibiotic concentration or the sudden pressure of the antibiotic indicates that sequential selection on the mediums containing kanamycin $25,50,75$ and $100 \mathrm{mg} / \mathrm{L}$ leads to the production of more roots and provides more varieties, while the sudden pressures with kanamycin 100 severely reduces the density of the roots in each explant.
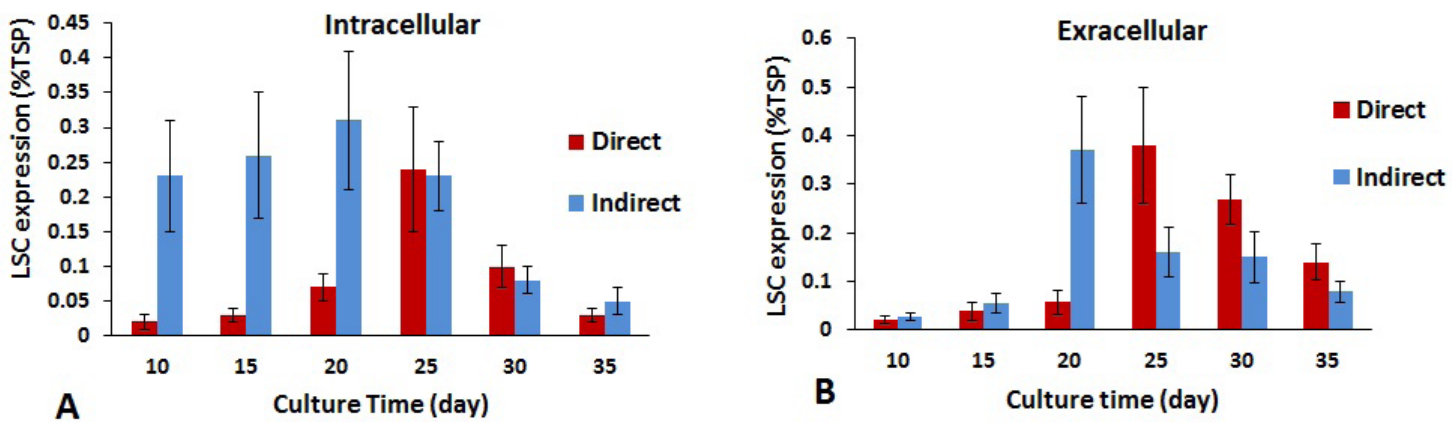

Figure 8. Measuring the Amount of LSC Recombinant Protein at Different Times in the Cell Extract (A) and in the Culture Medium (B) of the Hairy Root of Tobacco Plant in 2 Direct and Indirect Methods. 
It seems that the sudden pressure of antibiotics destroys the cells rapidly and releases acidic and phenolic substances that lead to the destruction of probably transformed cells, and results in fewer lines of the hairy root. This is while in the gradual increase of the selected factor, lower cell death occurs in the early stages, and a more favorable condition will be provided for the expression of the antibiotic-resistance gene in the transformed cells. In addition, the conducted studies also show the growth decline in high concentrations. ${ }^{28}$

The qualitative evaluation of GUS showed that the reporter gene expression is higher in the indirect method. The quantitative evaluation of GUS indicates that its expression in the indirect method was more than 7 times greater than in the direct method. In the indirect method, the transgenic sample selection was performed in 2 steps, first in the regeneration of transgenic plant and then in the induction of hairy root, so the hairy root developed from a transgenic plant with the adequate expression of antibiotic resistance gene (which is found in the same T-DNA with the gene of interest). While in the direct method, a mosaic of cells can occur among which some cells only have the root-inducing gene $(\mathrm{rolB})$ and the other cells receive both genes (gus and rolB genes), so the resulted hairy roots will be heterogeneous. On the other hand, a root may be selected in which the target gene has not been entered to a proper position, despite the fact that the rooting genes have entered to a good position. This means that there may even be a good root growth, but the target gene expression is weak. These genetically different hairy roots are selected as a transfromant while no difference can be detected in the initial roots. The results of the conducted studies also confirmed the superiority of the indirect method..$^{29}$ Another study showed that hairy roots could produce 1 to 7 times more recombinant protein in comparison to the original transgenic plants. ${ }^{3}$

Considering the difference between qualitative and quantitative expression of GUS reporter gene in these two methods, expression evaluation of another recombinant protein (LSC) was considered. Based on our results, the maximum expression of the intracellular recombinant protein in different lines of hairy root in the indirect method is $0.37 \%$ TSP which is 1.5 times higher than the direct method $(0.23 \%$ TSP). Another study has shown that expression in the hairy root is six and seven times more than its production in the leaf and root of the transgenic plant respectively which can be due to the more synthesis of protein in the hairy root. ${ }^{30}$

The study of the secreted protein into the culture medium in the direct and indirect method indicates that the amount of secreted protein into the medium (on days 25 and 20) is the same, however the amount of LSC produced into the hairy root cells in these two methods is significantly different. It seems that since in the design of the gene construct no specific mechanism has been used to secrete the recombinant protein out to the environment, the capacity of the plant to secrete the protein out of the cell is limited and does not depend on the amount of protein in the cell. ${ }^{31}$ According to a study, the expression of the recombinant ranalexin protein in transgenic plants was higher in the case of using the secretory signal peptide. ${ }^{32,33}$

The highest level of the recombinant protein expression in the direct method was found on day 25 and in the indirect method on day 20. These findings are in accordance with the previous reports about the expression of the recombinant protein in the hairy roots. After 25 days, the hairy root culture is still alive, but the expression level is significantly reduced, which is probably due to the decrease in the stability of enzymes and the reduction of translation. ${ }^{33}$ Also, extracellular factors such as loss of nutrients, significant increase in the proteases in the culture medium, and increase of the harmful substances due to cellular destruction can be referred, as in a ranalexin protein expression study was reported. ${ }^{32}$ The secreted recombinant protein into the culture medium can be collected by continuous replacement of the culture medium. In addition, protein stabilizers can be used to stabilize the secreted proteins. ${ }^{30}$

\section{Conclusions}

Comparing these 2 methods indicated that the hairy roots in the indirect method produce higher recombinant protein content than the direct method. Also, it seems that despite a longer tissue culture process, indirect hairy root induction method is a more suitable method for achieving greater recombinant protein expression.

\section{Authors' Contributions}

All authors equally contributed to the current study.

\section{Conflict of Interest Disclosures}

The authors declare they have no conflicts of interest.

\section{Acknowledgments}

Authors gratefully acknowledge the members of National Institute of Genetic Engineering and Biotechnology for the assistance.

\section{References}

1. De Muynck B, Navarre C, Boutry M. Production of antibodies in plants: status after twenty years. Plant Biotechnol J. 2010;8(5):529563. doi:10.1111/J.1467-7652.2009.00494.X.

2. Horn ME, Woodard SL, Howard JA. Plant molecular farming: systems and products. Plant Cell Rep. 2004;22(10):711-720. doi:10.1007/S00299-004-0767-1.

3. Skarjinskaia M, Ruby K, Araujo A, Et AL. Hairy roots as a vaccine production and delivery system. Adv Biochem Eng Biotechnol. 2013;134:115-134. doi:10.1007/10_2013_184.

4. Tiwari S, Verma PC, Singh PK, Tuli R. Plants as bioreactors for the production of vaccine antigens. Biotechnol Adv. 2009;27(4):449467. doi:10.1016/J.biotechadv.2009.03.006.

5. Guarino A, Dupont C, Gorelov Av, Et AL. The management of acute diarrhea in children in developed and developing areas: from evidence base to clinical practice. Expert Opin Pharmacother. 2012;13(1):17-26. doi:10.1517/14656566.2011.634800.

6. Beddoe T, Paton AW, Le Nours J, Rossjohn J, Paton JC. Structure, biological functions and applications of the AB5 toxins. Trends Biochem Sci. 2010;35(7):411-418. doi:10.1016/J. tibs.2010.02.003.

7. Tamamura Y, Tanaka K, Uchida I. Characterization of pertussislike toxin from Salmonella spp. that catalyzes ADP-ribosylation of G proteins. Sci Rep. 2017;7(1):2653. doi:10.1038/S41598-01702517-2.

8. Melton-Celsa A, Mohawk K, Teel L, O'brien A. Pathogenesis of shiga-toxin producing Escherichia coli. Curr Top Microbiol 
Immunol. 2012;357:67-103. doi:10.1007/82_2011_176.

9. Ghosh-Banerjee J, Senoh M, Takahashi T, et al. Cholera Cholera toxin production by the El Tor variant of Vibrio cholerae O1 compared to prototype El Tor and classical biotypes. J Clin Microbiol. 2010;48(11):4283-4286. doi:10.1128/Jcm.00799-10.

10. Allen KP, Randolph MM, Fleckenstein JM. Importance of heatlabile enterotoxin in colonization of the adult mouse small intestine by human enterotoxigenic Escherichia coli strains. Infect Immun. 2006;74(2):869-875. doi:10.1128/lai.74.2.869-875.2006.

11. Johannes L, Romer W. Shiga toxins--from cell biology to biomedical applications. Nat Rev Microbiol. 2010;8(2):105-116. doi:10.1038/Nrmicro2279.

12. Karaman S, Cunnick J, Wang K.Expression of the cholera toxin $B$ subunit (CT-B) in maize seeds and a combined mucosal treatment against cholera and traveler's diarrhea. Plant Cell Rep. 2012;31(3):527-537. doi:10.1007/S00299-011-1146-3.

13. Gu J, Liu Y, Yu S, Et Al. Enterohemorrhagic Escherichia coli trivalent recombinant vaccine containing EspA, intimin and Stx2 induces strong humoral immune response and confers protection in mice. Microbes Infect. 2009;11(10-11):835-841. doi:10.1016/J. micinf.2009.04.024

14. Oloomi M, Bouzari S, Emami S. A recombinant hybrid peptide composed of AAF adhesin of enteroaggregative Escherichia coli and Shiga toxin B subunit elicits protective immune response in mice. Eur J Clin Microbiol Infect Dis. 2009;28(11):1311-1316. doi:10.1007/S10096-009-0781-X.

15. Rosales-Mendoza S, Alpuche-Solis AG, Soria-Guerra RE, et al. Expression of an Escherichia coli antigenic fusion protein comprising the heat labile toxin B subunit and the heat stable toxin, and its assembly as a functional oligomer in transplastomic tobacco plants. Plant J. 2009;57(1):45-54. doi:10.1111/J.1365313X.2008.03666.X.

16. Yan J, Wang Y, Shao SH, Mao YF, Li HW, Luo YH. Construction of prokaryotic expression system of ItB-ureB fusion gene and identification of the recombinant protein immunity and adjuvanticity. World J Gastroenterol. 2004;10(18):2675-2679.

17. Kazemi R, Akhavian A, Amani J, et al. Immunogenic properties of trivalent recombinant protein composed of B-subunits of LT, STX-2, and CT toxins. Microbes Infect. 2016;18(6):421-429. doi:10.1016/J.micinf.2016.03.001.

18. Sambrook J, Russell DW. Molecular Cloning: A Laboratory Manual (3-Volume Set). Cold Spring Harbor Laboratory Press; 2001:2100.

19. Nilsson O, Crozier A, Schmulling T, Sandberg G, Olsson O. Indole3 -acetic acid homeostasis in transgenic tobacco plants expressing the Agrobacterium Rhizogenes Rolb gene. Plant J. 1993;3(5):681689. doi:10.1111/J.1365-313X.1993.00681.X.
20. Gallois P, Marinho P. Leaf disk transformation using Agrobacterium Tumefaciens-expression of heterologous genes in tobacco. Methods Mol Biol. 1995;49:39-48. doi:10.1385/0-89603321-X:39.

21. Amani J, Mousavi SL, Rafati S, Salmanian AH. Immunogenicity of a plant-derived edible chimeric EspA, Intimin and Tir of Escherichia coli O157:H7 in mice. Plant Sci. 2011;180(4):620627. doi:10.1016/J.plantsci.2011.01.004.

22. Terada R, Shimamoto K. Expression of CaMV35S-GUS gene in transgenic rice plants. Mol Gen Genet. 1990;220(3):389-392. doi:10.1007/Bf00391743.

23. Blazquez M. Quantitative gus activity assay of plant extracts. CSH Protoc. 2007;2007:Pdb.prot4690. doi:10.1101/Pdb.prot4690.

24. Agostini E, Hernandez-Ruiz J, Arnao MB, Milrad SR, Tigier HA, Acosta M. A peroxidase isoenzyme secreted by turnip (Brassica napus) hairy-root cultures: inactivation by hydrogen peroxide and application in diagnostic kits. Biotechnol Appl Biochem. 2002;35(Pt 1):1-7.

25. Lau OS, Sun SS. Plant seeds as bioreactors for recombinant protein production. Biotechnol Adv. 2009;27(6):1015-1022. doi:10.1016/J.biotechadv.2009.05.005

26. Zhang BH, Liu F, Liu ZH, Wang HM, Yao CB. Effects of kanamycin on tissue culture and somatic embryogenesis in cotton. Plant Growth Regul.2001;33(2):137-149.doi:10.1023/A:1017563327264.

27. TzfiraT, CitovskyV. Agrobacterium-mediated genetic transformation of plants: biology and biotechnology. Curr Opin Biotechnol. 2006;17(2):147-154. doi:10.1016/J.copbio.2006.01.009.

28. Haddadi F, Aziz MA, Abdullah SN, Tan SG, Kamaladini H. An efficient Agrobacterium-mediated transformation of strawberry cv. Camarosa by a dual plasmid system. Molecules. 2015;20(3):36473666. doi:10.3390/Molecules20033647.

29. Medina-Bolivar F, Cramer C. Production of recombinant proteins by hairy roots cultured in plastic sleeve bioreactors. Methods Mol Biol. 2004;267:351-363. doi:10.1385/1-59259-774-2:351.

30. Sunil Kumar GB, Ganapathi TR, Srinivas L, Revathi CJ, Bapat VA. Expression of hepatitis B surface antigen in potato hairy roots. Plant Sci. 2006;170(5):918-925. doi:10.1016/J.plantsci.2005.12.015.

31. Mergulhao FJ, Monteiro GA. Secretion capacity limitations of the Sec pathway in Escherichia Coli. J Microbiol Biotechnol. 2004;14(1):128-133.

32. Aleinein RA, Schafer $H$, Wink M. Rhizosecretion of the recombinant antimicrobial peptide ranalexin from transgenic tobacco hairy roots. J Bot Sci. 2015;1:45-55.

33. Woods RR, Geyer BC, Mor TS. Hairy-root organ cultures for the production of human acetylcholinesterase. BMC Biotechnol. 2008;8:95. doi:10.1186/1472-6750-8-95. 